\title{
Caracterización molecular de las comunidades bacterianas asociadas a sedimentos de un sistema costero del norte de la corriente de Humboldt, bahía de Mejillones del Sur, Chile
}

\author{
Molecular characterization of bacterial communities associated to sediments in the northern \\ Humboldt Current system, Mejillones del Sur bay, Chile
}

\author{
Rubén Araya ${ }^{1,2}$, Juan C. Leiva ${ }^{3}$ y Jorge Valdés ${ }^{4}$ \\ ${ }^{1}$ Instituto de Recursos Naturales Renovables (IA-RnR), Universidad de Antofagasta, Avda. Angamos 601, Antofagasta Chile \\ ${ }^{2}$ Departamento de Acuicultura, Universidad de Antofagasta, Avda. Angamos 601, Antofagasta Chile \\ ${ }^{3}$ Programa Doctorado en Ciencias Aplicadas Mención SMC, Universidad de Antofagasta, Avda. Angamos 601, Antofagasta, Chile \\ ${ }^{4}$ Instituto de Investigaciones Oceanológicas, Universidad de Antofagasta, Avda. Angamos 601, Antofagasta, Chile \\ raraya@uantof.cl
}

\begin{abstract}
To assess the structure of bacterial communities associated to coastal marine sediments of the Humboldt Current System of Mejillones bay $\left(23^{\circ} \mathrm{S}\right)$, Chile, we examined $16 \mathrm{~S}$ rDNA fragments of Bacteria Domain by denaturing gradient gel electrophoresis (DGGE), along with multidimensional scaling analysis (MDS). Sediments were taken from four sampling stations located at the bay and samples from four different depth points were analyzed for three periods during one year. The genetic analysis of prokaryont communities revealed a high bacterial diversity product indicated by the high number of bands detected by DGGE. The MDS analysis showed changes among the bacterial communities in all sampling stations, particularly on the community structures of prokaryotic cells associated to sediments at $10 \mathrm{~m}$, although we also found subtle differences in the structure of prokaryotic communities in all the depth analyzed. The findings of changes in the structure communities of prokaryotic cells could be triggered by changes in circulation water patterns inside the bay, which could be related to upwelling waters found at the northeast margin of the bay. In conclusion, the use of DGGE technique allowed us to establish bacterial community changes by qualitative analysis of samples obtained from different sampling stations at different periods.
\end{abstract}

Key words: DGGE, MDS, bacteria

\section{Introducción}

La corriente de Humboldt (HCS) es uno de los sistemas marinos más ricos en productividad biológica, lo que le permite sustentar una de las mayores producciones pesqueras del planeta (Summerhayes et al. 1995, González et al. 1998, Iriarte et al. 2000). Esta riqueza principalmente se debe al efecto fertilizador de aguas profundas que emergen hacia la superficie conducida por los vientos, las cuales aportan nutrientes a la zona eufótica sobre el margen del continente (Barber \& Smith 1981).
Resumen.- Para conocer la estructura de comunidades bacterianas asociadas a sedimentos marinos del sistema costero de la corriente de Humboldt en la bahía de Mejillones del Sur $\left(23^{\circ} \mathrm{S}\right)$, Chile, se realizaron análisis de electroforesis en gel de gradiente desnaturalizante (DGGE) a partir de fragmentos del 16S rADN para el Dominio Bacteria, junto con el análisis de escala multidimensional (MDS). Para esto, se realizaron muestreos de sedimentos en cuatro sectores dentro de la bahía de Mejillones del Sur, durante tres períodos de muestreo y cuatro profundidades. El análisis genético de las comunidades procarióticas estudiadas reveló una alta diversidad bacteriana, la cual se estableció por el gran número de bandas detectadas por DGGE. El análisis de MDS evidenció cambios en la estructura de las comunidades asociadas a los sedimentos recolectados a $10 \mathrm{~m}$, entre las 4 estaciones de muestreo. Se encontraron además, diferencias en la estructura de comunidades procarióticas asociadas a las distintas profundidades analizadas. Esta variación en la estructura genética de las comunidades procarióticas estuvo influenciada por cambios en los patrones de circulación al interior de la bahía, los cuales se asociarían al efecto de los focos de surgencia que ingresan por el margen noreste de la bahía. En conclusión, la técnica de DGGE nos permitió establecer cambios en la estructura de las comunidades bacterianas a través del análisis cualitativo de muestras obtenidas en los diferentes sectores y tiempos de muestreo.

Palabras clave: DGGE, MDS, bacteria

Esta particular característica a menudo genera ciertos cambios puntuales biológicos y oceanográficos, los cuales han sido ampliamente estudiados en los últimos años (Langue \& Berger 1993, Strub et al. 1998, Escribano \& Hidalgo 2000, Sobarzo \& Figueroa 2001, Blanco et al. 2001). Sin embargo, estos estudios se han enfocado principalmente en los ambientes pelágicos a una escala regional, dejando en un segundo plano los sistemas marinos costeros de escala local como lo son los ambientes de bahía. En Chile, la información para estos ambientes cubre aspectos tales como circulación de masas 
de agua, productividad biológica, impactos ambientales, manejo de recursos y ecología de comunidades. Sin embargo, no todas las bahías han sido estudiadas con igual intensidad, generándose vacíos de conocimiento que dificultan el entendimiento y comparación de los procesos propios de estos sistemas, así como de medidas administrativas que permitan un manejo armónico de dichos ambientes.

Uno de los aspectos menos estudiados en los ambientes costeros de Chile, es el relacionado con la estructura y dinámica de las comunidades microbiológicas que allí habitan; esto porque generalmente las bacterias han sido consideradas como una 'caja negra' dentro de los sistemas ecológicos (Kenzaka et al. 1998) y porque persistían deficiencias metodológicas que impedían un estudio representativo de estas comunidades de microorganismos (Wagner et al. 1993). Recientemente, el desarrollo de técnicas no dependientes de cultivo destinadas a evitar el bajo porcentaje de cultivabilidad (<1\%) (Amann et al. 1995, Muyzer et al. 1998) respecto de la fracción total de bacterias observables en muestras ambientales (Sahm \& Berninger 1998, Ellis et al. 2003), ha permitido conocer la variabilidad espacial y temporal de las comunidades (Ward et al. 1995). Además, el uso de herramientas moleculares como la técnica de electroforesis en gel de gradiente desnaturalizante (DGGE) (Muyzer et al. 1993), a partir de fragmentos de 16S rADN amplificados mediante la técnica de reacción en cadena de la polimerasa (PCR) (Mullis \& Faloona 1987), ha sido utilizada junto al análisis de escala multidimensional (MDS) para analizar poblaciones bacterianas asociadas a diversos ambientes como suelos y sedimentos (Brinkhoff et al. 1998, Rosado et al. 1998, Bruns et al. 1999, Traian et al. 2008), así como bacterioplancton de ambientes lacustres y marinos (Murray et al. 1998, Dumestre et al. 2001, Schafer et al. 2001, Casamayor et al. 2002).

La península de Mejillones ha sido descrita como el accidente geográfico más importante de la costa chilena, dando origen, en la región de Antofagasta, a dos grandes bahías con aperturas opuestas como son San Jorge y Mejillones del Sur (Vargas 2002). Particularmente, esta bahía, pertenece al sistema de surgencias de Punta Angamos, descrito como el sistema de surgencias más productivo de la zona norte de Chile (Escribano 1998, González et al. 1998, Marín \& Olivares 1999, Escribano \& Hidalgo 2000, Thomas et al. 2001, Sobarzo \& Figueroa 2001), con valores de productividad primaria que alcanzan los $1.070 \mathrm{~g} \mathrm{C} \mathrm{m}^{-2} \mathrm{y}^{-1}$ (Marín et al. 1993). La circulación al interior de la bahía se caracteriza por el ingreso de aguas de surgencias por el margen noreste y por el noreste lo que genera un giro ciclónico en el centro de la bahía, con una clara estratificación térmica del agua (Escribano et al. 2002, Marín et al. 2003). Estas características influyen fuertemente en el comportamiento estacional de la bahía, caracterizada por una estación fría y una cálida, donde los pulsos de surgencias conducen al continuo flujo de material orgánico hacia el fondo de la bahía. Sin embargo, poco se conoce respecto de cómo se comportan o influyen estacionalmente las características oceanográficas sobre las comunidades bacterianas asociadas a los sedimentos de la bahía de Mejillones del Sur. Por tal motivo, el objetivo del presente estudio fue describir cambios en la estructura genética de comunidades bacterianas asociadas a sedimentos marinos de la bahía de Mejillones del Sur. Para esto, se seleccionaron a lo largo de la costa, cuatro transectas de muestreo, las cuales fueron analizadas a partir de sedimentos obtenidos desde distintas profundidades mediante la técnica de electroforesis en gel de gradiente desnaturalizante (DGGE). Este es el primer reporte en la literatura nacional acerca de la utilización de esta técnica de caracterización molecular, en especial, para una zona en la cual coexisten diversas actividades humanas como acuicultura, pesquería, generación de energía y servicios asociados a la minería.

\section{Material y métodos}

Obtención de muestras de sedimentos y determinación de parámetros físico-químicos en la columna de agua

Los sedimentos fueron recolectados con un box-core de $225 \mathrm{~cm}^{2}$ de superficie, a partir de cuatro transectas perpendiculares a la línea de costa, localizadas en distintos puntos de la bahía de Mejillones, $\left(23,5^{\circ} \mathrm{S}\right)$, e individualizadas como T1: Punta Chacaya, T2: Industrial, T3: Capitanía y T4: Punta Rieles (Fig. 1). Desde cada transecta se recolectaron muestras de sedimentos en triplicado a partir de sucesivos dragados a $10 \mathrm{~m}, 25 \mathrm{~m}$, $50 \mathrm{~m} \mathrm{y} 75 \mathrm{~m}$ de profundidad. Cada muestra fue almacenada en frascos plásticos estériles de $50 \mathrm{~mL}$ de capacidad (Corning) y mantenidas a $4^{\circ} \mathrm{C}$ para su transporte al laboratorio de Microbiología de Sedimentos de la Universidad de Antofagasta. Este procedimiento se repitió tres veces; en septiembre 2005, diciembre 2005 y mayo 2006. Adicionalmente, al momento del muestreo, se registraron las variables físico-químicas de la columna de agua como temperatura, oxígeno disuelto y clorofila, por medio de una sonda multiparámetro YSY modelo 6600 y un CTD SeaBird 19 Plus. El análisis de materia orgánica se realizó mediante la metodología descrita por Nelson \& Sommers (1982).

\section{Extracción de ADN y purificación}

Las muestras de sedimentos obtenidas por dragado, en triplicado, fueron mezcladas en partes iguales para posteriormente extraer el ADN genómico de las bacterias siguiendo la metodología descrita por Tsai \& Olson (1991). 


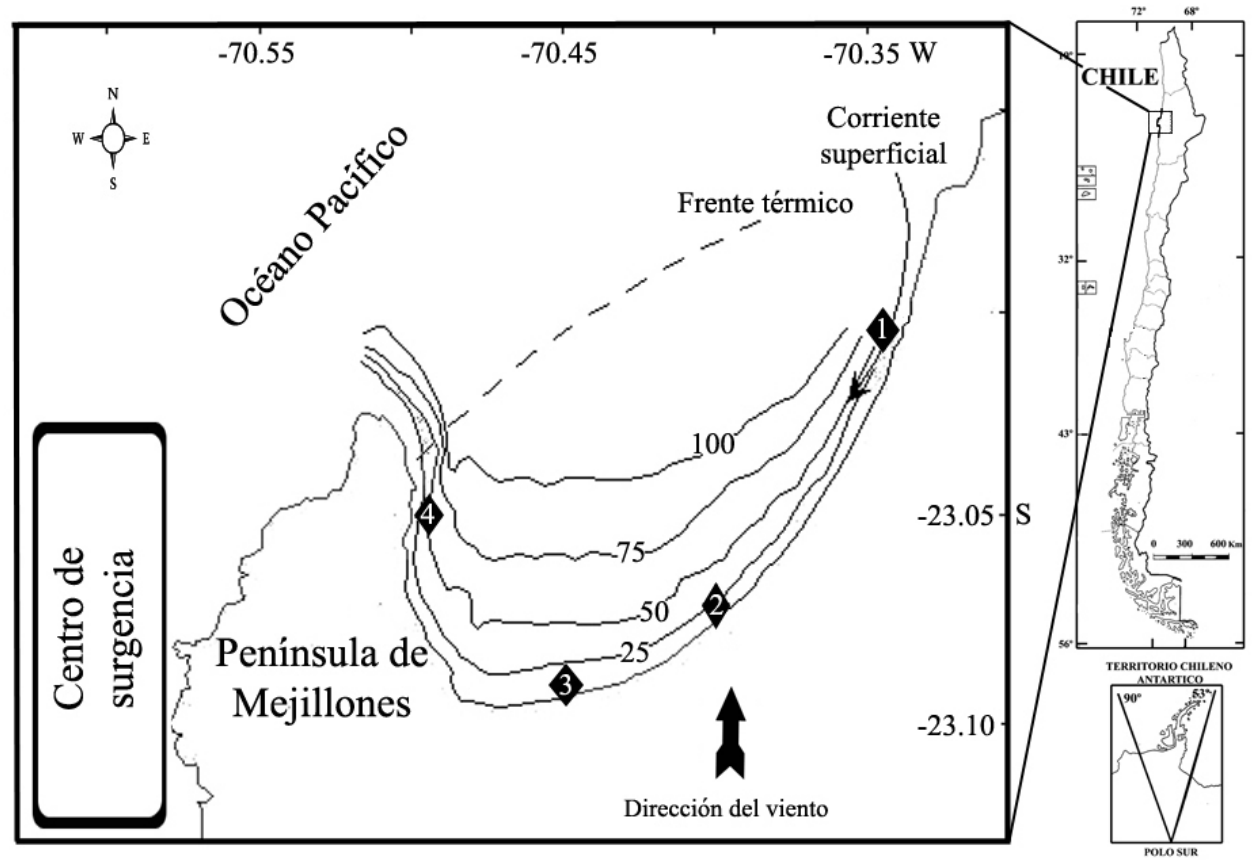

Figura 1

Mapa de las transectas de muestreo localizadas en la bahía de Mejillones del Sur, Chile

Map of the sampling stations in Mejillones del Sur bay, Chile

Tabla 1

Partidores utilizados para la amplificación de fragmentos de 16S rADN

Primers used for the amplification of $16 \mathrm{~S}$ rDNA fragments

\begin{tabular}{lcll}
\hline \multicolumn{1}{c}{ Oligonucleótido } & Posición & \multicolumn{1}{c}{ Target } & \multicolumn{1}{c}{ Secuencia } \\
\hline EUB f933 & $933-954$ & Bacteria regions & 5"-GC-clamp-GCACAAGCGGTGGAGCATGTGG-3" \\
& & V6-V8 & \\
EUB r1387 & $1387-1368$ & Bacteria regions & 5"-GCCCGGGAACGTATTCACCG-3" \\
& & V6-V8 & 5"-CGCCCGCCGCGCGCGGCGGGCGGGGCGGGGGCACGGGGGG \\
\hline
\end{tabular}

* Para análisis de DGGE se sintetizó en la posición 5'-end una abrazadera rica en GC.

Para esto, las bacterias asociadas a $1 \mathrm{~g}$ de muestra de sedimento fueron lisadas por medio de lisozima (Lyzozyme, Sigma Chemical Co.) y fraccionamiento físico, por medio del congelamiento y calentamiento ('freeze-thaw'). Posteriormente, el ADN fue extraído con dodecyl sulfato sódico (SDS) y fenol-cloroformo, para posteriormente ser precipitado con etanol al $100 \%$ a $-20^{\circ} \mathrm{C}$ durante $12 \mathrm{~h}$. Las moléculas de ARN fueron removidas por medio de la incubación con ARNasa A $\left(0,2 \mu \mathrm{g} \mu \mathrm{L}^{-1}\right)$ desde los extractos crudos. Posteriormente, el ADN libre de ARN fue purificado con una columna Elutip-d (Scheicher \& Schuell, Keene, N.H., U.S.A.), con un prefiltro Scheicher \& Schuell NA010/ $27(0,45 \mu \mathrm{m}$, acetato de celulosa). Por último el ADN fue recuperado desde la columna siguiendo las instrucciones del fabricante.

\section{Partidores y amplificación por PCR}

Fragmentos de $16 \mathrm{~S}$ rADN fueron amplificados utilizando los partidores EUBf933-GC-clamp y EUBr1387 (Tabla 1), los cuales son específicos para la secuencia conservada del $16 \mathrm{~S}$ rADN del dominio Bacteria (Iwamoto et al. 2000). Para prevenir la disociación completa de los fragmentos de ADN durante el análisis por DGGE, una abrazadera ('clamp') de 40-bp rica en GC fue incorporada en la posición 5' final del partidor EUBf933 (Myers et al. 1985). Las reacciones de amplificación se realizaron utilizando ADN polimerasa 
(AmpliTaq Gold; Applied Biosystem), en un volumen total de $50 \mu \mathrm{L}$ de la mezcla para PCR. Las concentraciones de los distintos componentes fueron $3 \mathrm{mM}$ de cloruro de magnesio y 20 pmol de cada partidor. El protocolo de PCR utilizado fue con 'hot start' a $95^{\circ} \mathrm{C}$ por 9 min, seguido de un 'touchdown': la temperatura de alineamiento se calibró inicialmente a $65^{\circ} \mathrm{C}$ y luego se redujo en $0,5^{\circ} \mathrm{C}$ por cada ciclo hasta llegar a $55^{\circ} \mathrm{C}$, seguida de una extensión de los partidores a $72^{\circ} \mathrm{C}$ por $3 \mathrm{~min}$. Posteriormente, se realizaron 15 ciclos adicionales a $55^{\circ} \mathrm{C}$ por $1 \mathrm{~min}$ (alineamiento de los partidores), con una desnaturación a $94^{\circ} \mathrm{C}$, por 1 min y extensión de los partidores a $72^{\circ} \mathrm{C}$, por $3 \mathrm{~min}$. Finalmente, se realizó un paso de extensión a $72^{\circ} \mathrm{C}$ por $7 \mathrm{~min}$.

\section{Análisis por electroforesis en gel de gradiente desnaturalizante (DGGE)}

Aproximadamente $300 \mathrm{ng}$ de los productos obtenidos por PCR fueron cargados sobre un gel de poliacrilamida $(6,5 \% \mathrm{p} / \mathrm{v})$, conteniendo un gradiente de desnaturalización del $45 \%$ al $65 \%$ (urea-formamida). La electroforesis fue llevada a cabo a $55^{\circ} \mathrm{C}$ por $12 \mathrm{~h}$ a $100 \mathrm{~V}$ en una solución buffer de 1x TAE. Luego, los geles se tiñeron por $20 \mathrm{~min}$ con SYBR Gold (nucleic acid, Molecular Probes) siguiendo las instrucciones del fabricante. Los geles de DGGE fueron digitalizados por medio de un transiluminador (Vilber Lourmat, modelo TFX20M) con cámara digital (Q-Imaging modelo micropublisher 3.3). Las imágenes de los geles se analizaron para generar un perfil densitométrico de las bandas mediante el programa GelPro Analyzer para Windows (v.4.0.00.00.001, Media Cibernetic), el cual fue exportado a una planilla de datos Microsoft Excel v.2003 para Windows. La detección de las bandas fue registrada cuando la cumbre de éstas excedió el $1 \%$ del total de los picos de la gráfica. Para evitar el efecto de bandas no reproducibles o desviaciones de PCR (sesgo de PCR), se compararon amplificaciones paralelas de PCR de la misma muestra, obteniéndose perfiles de DGGE similares. Para el análisis de la comunidad bacteriana, los datos desde la planilla Excel fueron utilizados con el programa Primer 6 para Windows (v 6.1.6, Primer-E Ltda), para construir diagramas de agregación mediante media de grupos y escalamiento métrico multidimensional (MDS); para ello se utilizó la matriz de similitud de Bray \& Curtis (1975).

\section{Resultados}

\section{Análisis de bandas obtenidas por DGGE a través de MDS y análisis de agrupamiento}

El análisis genético por medio de DGGE para las distintas transectas analizadas se muestra en la Fig. 2, a partir de cuatro geles que cubren un total de 43 muestras de sedimentos obtenidos en distintas profundidades y períodos de muestreo. El análisis de estos geles, a partir de la generación de perfiles densitométricos de bandas, resultó en la detección total de 840 bandas, las cuales fueron registradas cuando la cumbre de éstas excedió el $1 \%$ del total de los picos de la gráfica (Tabla 2). El promedio de bandas para todos los sectores analizados fue de $19,5 \pm 6,1$, las que variaron entre 7 bandas para el sector Industrial (T2) a una profundidad de $10 \mathrm{~m}$, en septiembre de 2005, hasta 32 bandas para el sector Punta Chacaya (T1), a una profundidad de $50 \mathrm{~m}$, en diciembre de 2005. Los cambios espaciales y temporales en la estructura de las comunidades revelaron que los patrones de bandas de los sedimentos obtenidos desde los $10 \mathrm{~m}$ de profundidad en septiembre de 2005, en Punta Chacaya (T1) e Industrial (T2), presentaron una estructura de comunidad diferente a los perfiles obtenidos para sedimentos recolectados a $\operatorname{los} 25,50$ y $75 \mathrm{~m}$ de profundidad (Fig. 3a y b). Coincidentemente, la construcción de clústeres para estos patrones de bandas, mostró valores de homogeneidad del 60 y 50\% para las transectas T1 y T2, respectivamente (Fig. 3a y b). Por el contrario, el análisis de las transectas Capitanía (T3) y Punta Rieles (T4) mostró, tanto por MDS como por el análisis de agrupamiento, la presencia de 3 y 2 clústeres, respectivamente.

\section{Análisis de variables físico-químicas}

El análisis de variables físico-químicas se observa en la Fig. 4. Los perfiles de temperatura para las distintas transectas al momento de los muestreos de sedimentos evidenciaron, en general, patrones homogéneos sin la presencia de una clara termoclina superficial en especial para el período de septiembre 2005 y mayo 2006. Sin embargo, para el período de diciembre 2005 la distribución de la temperatura en las distintas transectas fue homogénea, a excepción de la transecta 1 (Fig. 4a). Esta última evidenció una pequeña termoclina superficial a los $6 \mathrm{~m}$, para, a partir de esta profundidad, mantenerse homogénea en comparación de los perfiles registrados para las otras transectas (Fig. 4a).

Los perfiles de oxígeno disuelto en la columna de agua mostraron variaciones estacionales de este parámetro, en especial para septiembre 2005 y mayo 2006, con niveles de oxígeno por sobre los $5 \mathrm{mg} \mathrm{L}^{-1}$ para cada una de las transectas hasta los $21 \mathrm{~m}$, a excepción de la transecta 1 , la cual bajo los $13 \mathrm{~m}$ cayó con niveles inferiores a $2 \mathrm{mg}$ $\mathrm{L}^{-1}$. En diciembre 2005 se registró una disminución de este gas bajo los $6 \mathrm{mg} \mathrm{L}^{-1}$ a partir de los $10 \mathrm{~m} \mathrm{de}$ profundidad, para las transectas 1,3 y 4, a diferencia de la transecta 2 con niveles inferiores a $\operatorname{los} 2 \mathrm{mg} \mathrm{L}^{-1}$ a partir de los $6 \mathrm{~m}$ de profundidad (Fig. 4b). 

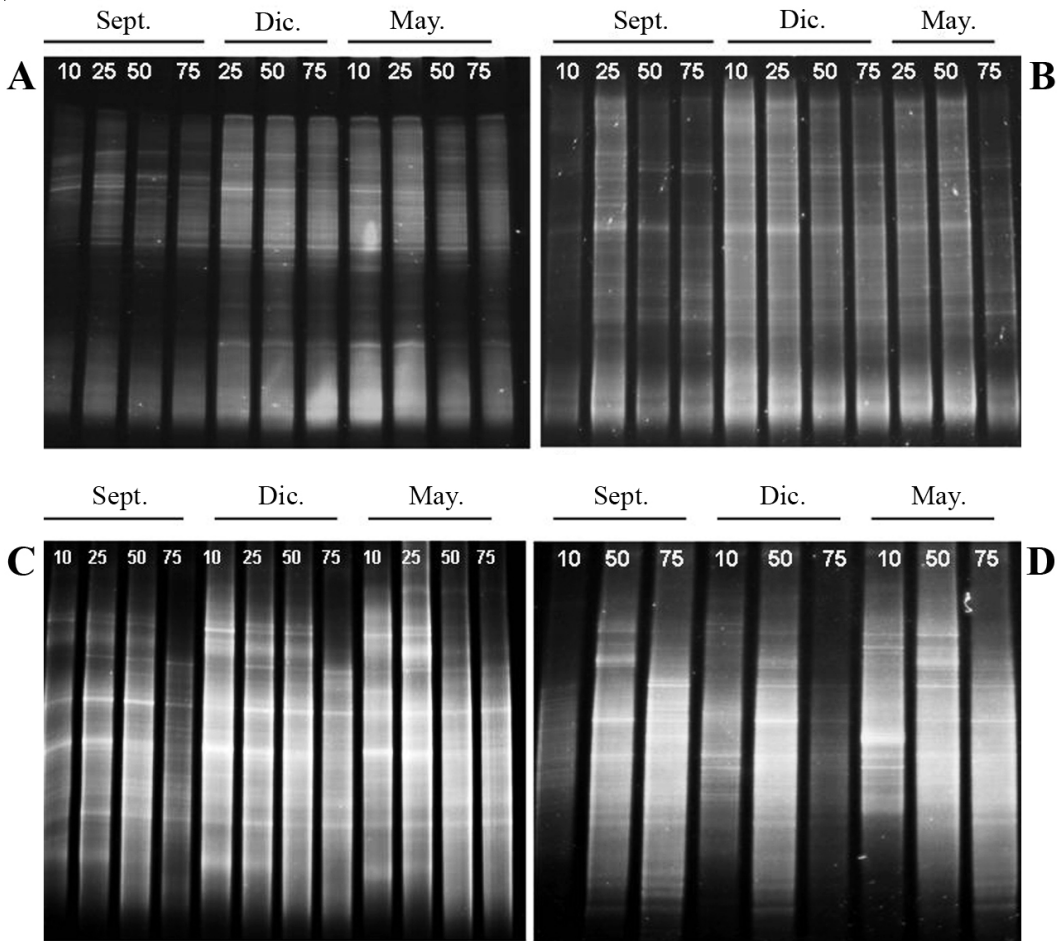

Figura 2

Visualización de geles de DGGE a partir de fragmentos del 16S rADN obtenidos por PCR para muestras recolectadas desde las transectas 1(A), 2(B), 3(C) y 4(D). El orden cronológico del análisis se expresa para septiembre 2005, diciembre 2005 y mayo 2006 para sedimentos obtenidos desde 10, 25, 50 y 75 m de profundidad

DGGE of PCR-amplified 16S rDNA fragments of sediments associated bacteria from different sample stations 1(A), 2(B), 3(C) and 4(D). The time course of the sediment samples analysis is indicated for September 2005,

December 2005 and May 2006 for sediments sampled at 10, 25, 50 and $75 \mathrm{~m}$ deep

Tabla 2

Número de bandas detectadas por medio de electroforesis en gel de gradiente desnaturalizante (DGGE) desde las distintas transectas analizadas en tres períodos estacionales

Number of detected bands by the use of denaturing gradient gel electrophoresis (DGGE) from the different sampling stations along different seasonal periods

\begin{tabular}{|c|c|c|c|c|c|c|}
\hline \multirow[t]{2}{*}{ Profundidad (m) } & \multirow[t]{2}{*}{ Fecha } & \multicolumn{4}{|c|}{ Número de bandas } & \multirow{2}{*}{$\begin{array}{c}\text { Promedio } \\
\text { bandas }\end{array}$} \\
\hline & & $\mathrm{T} 1$ & $\mathrm{~T} 2$ & $\mathrm{~T} 3$ & $\mathrm{~T} 4$ & \\
\hline 10 & Sep-05 & 18 & 7 & 9 & 13 & 11,8 \\
\hline 25 & Sep-05 & 26 & 22 & 13 & NA & 20,3 \\
\hline 50 & Sep-05 & 24 & 15 & 13 & 25 & 19,3 \\
\hline 75 & Sep-05 & 24 & 17 & 15 & 25 & 20,3 \\
\hline Promedio bandas & & 23 & 15,3 & 12,5 & 21 & \\
\hline 10 & Dic-05 & NA & 18 & 10 & 26 & 18 \\
\hline 25 & Dic-05 & 24 & 19 & 16 & NA & 19,7 \\
\hline 50 & Dic-05 & 32 & 20 & 16 & 23 & 22,8 \\
\hline 75 & Dic-05 & 30 & 20 & 13 & 16 & 19,8 \\
\hline Promedio bandas & & 28,7 & 19,3 & 13,8 & 21,7 & \\
\hline 10 & May-06 & 27 & NA & 12 & 26 & 21,7 \\
\hline 25 & May-06 & 28 & 20 & 15 & NA & 21 \\
\hline 50 & May-06 & 29 & 19 & 13 & 22 & 20,8 \\
\hline 75 & May-06 & 26 & 20 & 14 & 20 & 20 \\
\hline Promedio bandas & & 27,5 & 19,7 & 13,5 & 22,7 & \\
\hline
\end{tabular}

NA : No Analizada
La clorofila total para cada una de las transectas analizadas para los distintos períodos del estudio, mostró grandes variaciones entre septiembre y diciembre 2005 (Fig. 4c). Se observaron claramente los bajos niveles de este pigmento durante el período de muestreo de septiembre 2005, cuando los niveles registrados fueron inferiores a $4 \mathrm{mg} \mathrm{L}^{-1}$ a partir de los $2 \mathrm{~m}$ de profundidad. Inversamente, durante diciembre 2005, los niveles de clorofila detectados para las transectas 3 y 4 incrementaron su concentración por sobre $6 \mathrm{mg} \mathrm{L}^{-1}$, a partir del nivel superficial hasta los $10 \mathrm{~m}$ de profundidad. Sin embargo, los perfiles obtenidos para las transectas 1 y 2 se mostraron homogéneos desde la superficie hasta los $16 \mathrm{~m}$ de profundidad (Fig. 4c).

\section{Análisis de materia orgánica}

La determinación de materia orgánica para los sedimentos recolectados desde cada una de las transectas durante los distintos períodos de muestreo se observan en la Fig. 5. 

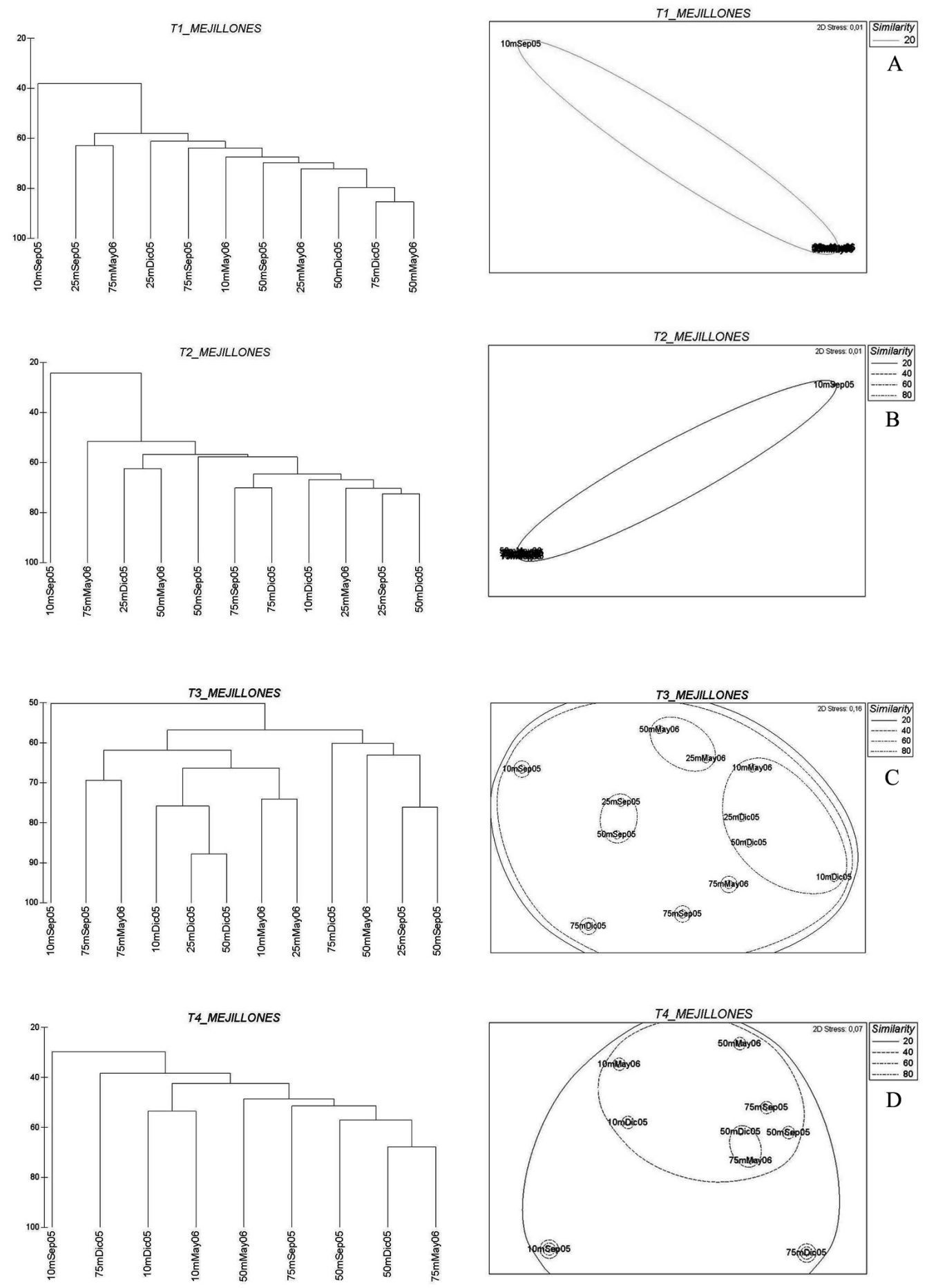

Figura 3

Análisis bidimensional por medio de MDS (columna derecha) y agrupamiento (columna izquierda) a partir de geles de DGGE para fragmentos del 16S rADN obtenidos por PCR para muestras recolectadas desde las transectas 1(A), 2(B), 3(C) y 4(D) durante septiembre 2005, diciembre 2005 y mayo 2006 para sedimentos obtenidos desde 10, 25, 50 y 75 m de profundidad

Two-dimensional plots of MDS (right column) and cluster (left column) analysis from DGGE patterns of sediment associated bacteria for sampling stations 1(A), 2(B), 3(C) and 4(D) along September 2005, December 2005 and May 2006

for sediments sampled at $10,25,50$ and $75 \mathrm{~m}$ deep 
Temperatura $\left({ }^{\circ} \mathrm{C}\right)$

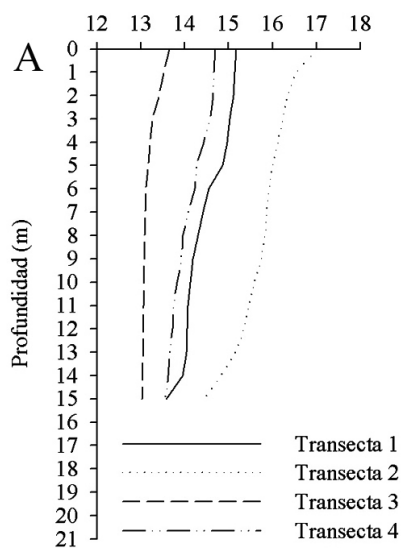

Oxígeno $\left(\mathrm{mLL}^{-1}\right)$

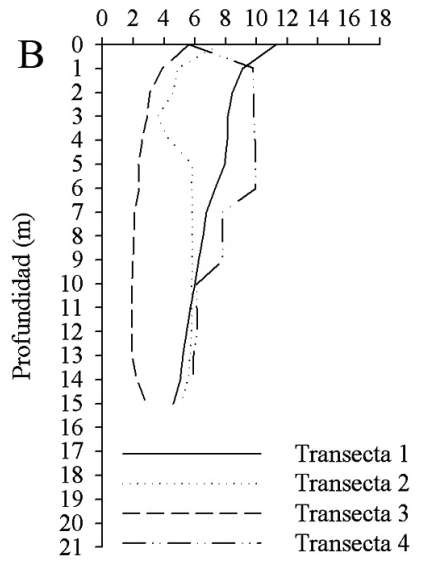

Clorofila total $\left(\mathrm{mg} \mathrm{L}^{-1}\right)$

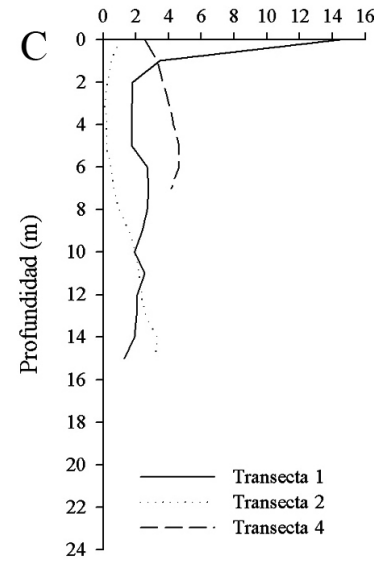

Temperatura $\left({ }^{\circ} \mathrm{C}\right)$

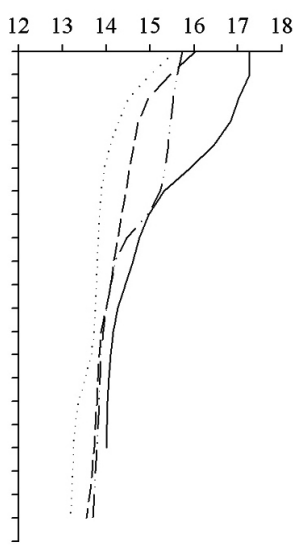

Oxígeno $\left(\mathrm{mLL}^{-1}\right)$

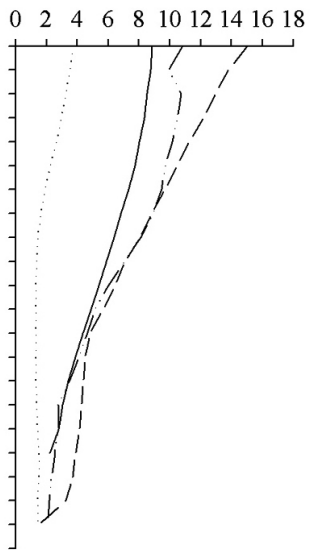

Clorofila total $\left(\mathrm{mg} \mathrm{L}^{-1}\right)$

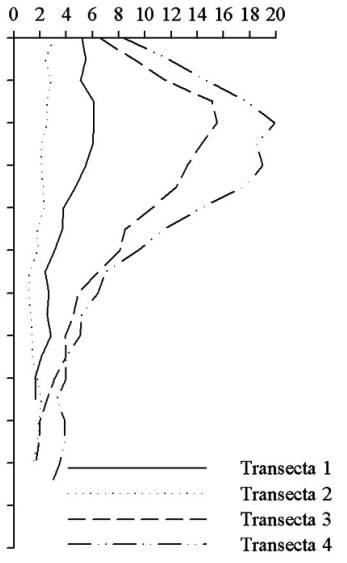

Figura 4
Temperatura $\left({ }^{\circ} \mathrm{C}\right)$

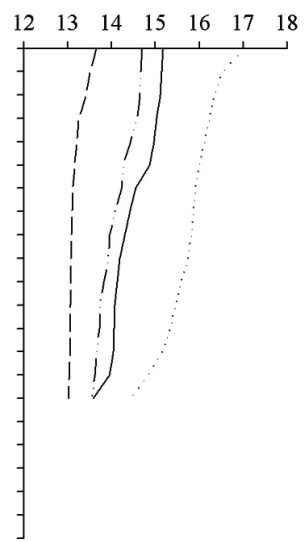

Oxígeno $\left(\mathrm{mLL}^{-1}\right)$

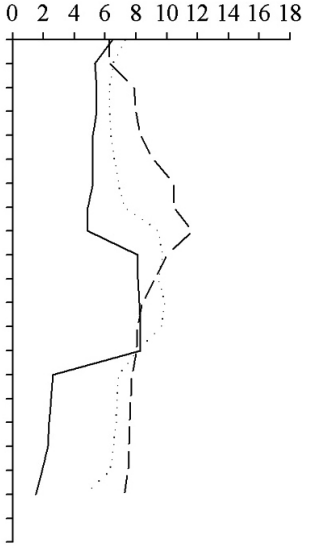

Perfiles de temperatura (A), oxígeno disuelto (B) y clorofila (C), registrados en la columna de agua para las distintas transectas,
durante septiembre 2005 (columna izquierda), diciembre 2005 (columna central) y mayo 2006 (columna derecha)

Temperature (A), disolved oxygen (B) and chlorophyll (C) patterns registered on seawater column for the different sampling stations during September 2005 (left column), December 2005 (central column) and May 2006 (right column) 

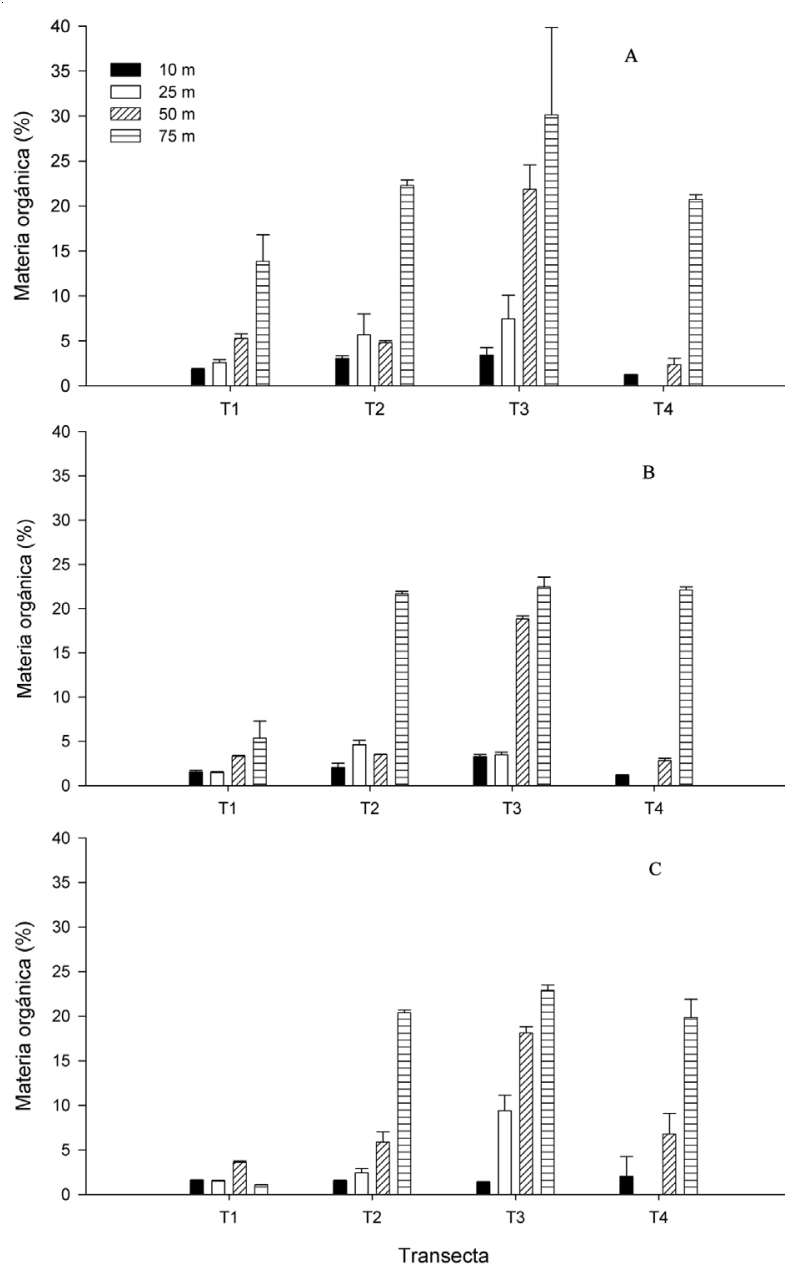

Figura 5

\section{Porcentaje de materia orgánica de sedimentos marinos recolectados desde las transectas $\mathrm{T} 1, \mathrm{~T} 2, \mathrm{~T} 3$ y $\mathrm{T} 4$ durante septiembre 2005 (A), diciembre 2005 (B) y mayo 2006 (C). Los valores representan el promedio del análisis de muestras en triplicado \pm desviación estándar}

Percentage of organic matter in marine sediments obtained from the sampling stations T1, T2, T3 and T4 during September 2005 (A), December 2005 (B) and May 2006 (C). Results represent the average of triplicate analyzed samples \pm standard deviation

Estos resultados muestran, en general, que sedimentos recolectados a profundidades inferiores a $25 \mathrm{~m}$ registraron niveles inferiores al 5\% de materia orgánica. A diferencia de esto, sedimentos mas profundos $(75 \mathrm{~m})$ mostraron valores de materia orgánica por sobre el $20 \%$, sin grandes variaciones a nivel estacional.

\section{Discusión}

El análisis genético espacial y temporal a partir de la utilización de DGGE, mostró en general un alto número de bandas para los distintos geles analizados. Si bien cada una de las bandas totales detectadas representaría una especie bacteriana (Muyzer et al. 1993), la diversidad ecológica así como la estructura de la comunidad de los sedimentos marinos de la bahía de Mejillones del Sur evidenciaron una alta y compleja diversidad procariótica. La variación en la estructura genética de las comunidades bacterianas observadas para los sedimentos recolectados a $10 \mathrm{~m}$ de profundidad en septiembre 2005 , desde las transectas $\mathrm{T} 1$ y T2, respecto de las transectas $\mathrm{T} 3$ y T4, reflejaría las diferentes condiciones que afectan a las comunidades microbianas. Como se ha señalado por Nelson et al. (2008) al estudiar comunidades bacterianas planctónicas marinas, cambios en la circulación de las aguas y de factores no tradicionalmente medidos por estudios oceanográficos, provocarían cambios estructurales en las comunidades procarióticas, como los observados en el presente estudio para las distintas transectas. Esto último se atribuye a que el movimiento de las aguas genera condiciones diferentes entre cada una de las transectas analizadas. De esta manera, el análisis de parámetros físico-químicos de la columna de agua reveló que en el caso de la temperatura, las diferencias son más evidentes al comparar las transectas entre sí, que al comparar los distintos tiempos de muestreo. Esto evidenciaría una mayor mezcla de la columna de agua para este período, debido a la entrada de masas de aguas provenientes del foco de surgencia que ingresarían por el margen noreste de la bahía, particularmente por el sector de la transecta 1 (Punta Chacaya). Este fenómeno influencia puntualmente el margen costero de los $10 \mathrm{~m}$ de profundidad, hasta el sector de Capitanía de Puerto (T3) frente a la ciudad de Mejillones. A diferencia de la entrada de masas de aguas con la trayectoria de un giro ciclónico por el sector de Punta Chacaya, el sector de Punta Rieles (T4) se caracteriza por presentar pulsos de surgencia que entran desde afloramientos en el sector de Punta Angamos. Esto último marcaría diferencias en los patrones de circulación y en la estructura de comunidades bacterianas asociada a los $10 \mathrm{~m}$ de profundidad en el período de septiembre 2005. Se ha descrito que los mayores pulsos de surgencia en el sistema de Punta Angamos y que influirían en la dinámica interna de la bahía de Mejillones, ocurriría principalmente cuando comienza el período de primavera (Marín \& Olivares 1999). En este período, el ingreso de aguas de surgencia de menor contenido de oxígeno disuelto, menor temperatura y mayor contenido de nutrientes, modifica la estructura de la columna de agua, provocando un ascenso de la zona mínima de oxígeno lo que hace que una mayor extensión del piso marino de la bahía presente 
ambientes de deposición pobres en oxígeno (Valdés et al. 2008). Como consecuencia de esto, se genera en la superficie un frente térmico que transforma la bahía en una sombra de surgencia con la consecuente retención de los nutrientes en superficie y una alta productividad primaria (Marín et al. 2003). Esto último se observa en los perfiles de clorofila, cuyas concentraciones son evidentemente mayores durante diciembre, correspondiendo a un período de alta productividad. La entrada de nutrientes a la bahía puede ser a través de los sectores T1 y T4 lo que produciría afloramientos durante septiembre, lo que consecuentemente incrementaría la diversidad genética de comunidades bacterianas. Por ejemplo, mediante la utilización de DGGE se ha observado que el ingreso de nutrientes al mar Mediterráneo produciría diferencias en el desarrollo y la estructura de las comunidades bacterianas (Schäfer et al. 2001, Lebaron et al. 2001). Esto podría incrementar la proliferación de poblaciones bacterianas fisiológicamente activas, generándose así diferencias en la estructura de las comunidades, lo cual ha sido reportado por Araya et al. (2003). Si bien el número de bandas detectadas en las estaciones T1 y T2 fue superior con 18 y 13 especies, respectivamente, en comparación con las 7 y 9 bandas observadas en el análisis de geles obtenidos desde las estaciones de muestreo T3 y T4 para el mismo período. Las diferencias entre la estructura de la comunidad de los sedimentos analizados a los $10 \mathrm{~m}$ respecto de los sedimentos recolectados a otras profundidades, podrían atribuirse a la aparición de nuevas bandas que no fueron detectadas en las otras muestras analizadas o también por efecto de sesgo asociado a la técnica del PCR. Esto es, diferencias en el número de copias de los genes o heterogeneidad del 16S rADN dentro de las especies. Si bien es cierto que la técnica de PCR presenta algunas desventajas, las técnicas genéticas de huella dactilar como PCR-DGGE y PCR-temperatura (T)GGE pueden clarificar los cambios en las comunidades microbianas evitando así la utilización de métodos que incluyan el cultivo bacteriano por medio de técnicas tradicionales (Santegoeds et al. 1998, Van Hannen et al. 1999, Schäfer et al. 2001, Traian et al. 2008).

La estabilidad relativa observada para las comunidades bacterianas obtenidas desde las transectas T1 y T4 a partir de bacterias asociadas a sedimentos por debajo de los $10 \mathrm{~m}$ de profundidad, podría ser una respuesta a una estabilidad mecánica de los sedimentos, los cuales no experimentarán bioturbación (Ortlieb et al. 2000) como en el área costera de los $10 \mathrm{~m}$ de profundidad. Estos sedimentos se caracterizan por poseer restos orgánicos depositados en la zona más somera de la bahía, los cuales corresponden a una mezcla de materia orgánica metabolizable y no metabolizable, de manera que se espera que ocurra una alta degradación de este último material debido a que son depositados en un ambiente con altos niveles de oxígeno (Valdés et al. 2005). Esto permite suponer que en el área costera de los 10 m existió una alta actividad bacteriana asociada a la transformación de la materia orgánica, lo que explicaría la diferencia en la estructura de las comunidades de estas muestras, respecto de la diversidad genética observada para los sedimentos de las otras muestras analizadas. En conclusión, los resultados observados en el presente estudio mostraron que los patrones de bandas obtenidos por DGGE y su análisis por medio de MDS o la construcción de árboles de agrupamiento ('clusters') fue discriminatorio de acuerdo a las interrogantes planteadas. Sin embargo, para entender los mecanismos que controlan la estructura y función de las comunidades bacterianas asociadas a sedimentos, se requiere de un análisis seriado en el tiempo que incluya el registro de un amplio número de parámetros físico-químicos y oceanográficos en el área de estudio.

\section{Agradecimientos}

Agradecemos el financiamiento del proyecto Innova Chile en su línea de financiamiento de Interés Público $\mathrm{N}^{\circ}$ 04CR7IXM-04. Los autores también agradecen a los evaluadores anónimos por los comentarios que contribuyeron a mejorar el presente manuscrito.

\section{Literatura citada}

Amann RI, W Ludwig \& K-H Schleifer. 1995. Phylogenetic identification and in situ detection of individual microbial cells without cultivation. Microbiological Reviews 59: 143-169.

Araya R, K Tani, T Takagi, N Yamaguchi \& M Nasu. 2003. Bacterial activity and community composition in stream water and biofilm from an urban river determined by fluorescent in situ hybridization and DGGE analysis. FEMS Microbiology Ecology 43(1): 111-119.

Barber R \& R Smith. 1981. Coastal upwelling ecosystems. En: Longhurst AR (ed). Analysis of marine ecosystems, pp. 3168. Academic Press, Londres.

Blanco JL, AC Thomas, ME Carr \& PT Strub. 2001. Seasonal climatology of hydrographic conditions in the upwelling region off Northern Chile. Journal of Geophysical Research Oceans 106: 11451-11467.

Bleck J. 1998. Estructura comunitaria de los ensambles zooplanctónicos epipelágicos del norte de Chile durante el desarrollo del evento “'El Niño" 1991-1992. Tesis de Biología Marina, Universidad Arturo Prat, Iquique, 39 pp.

Bray JR \& JT Curtis. 1975. An ordination of the upland forest communities of southern Wisconsin. Ecological Monographs 27: 325-349.

Brinkhoff T, CM Santegoeds, K Sahm, J Kuever \& G Muyzer. 1998. A poyphasic approach to study the diversity and vertical distribution of sulfur-oxidizing thiomicrospira species in 
coastal sediments of the german Wadden sea. Applied and Environmental Microbiology 64: 4650-4657.

Bruns MA, JR Stephen, GA Kowalchuk, JI Prosser \& EA Paul. 1999. Comparative diversity of ammonia oxidizer $16 \mathrm{~S}$ rRNA gene sequences in native, tilled, and successional soils. Applied and Environmental Microbiology 65: 2994-3000.

Casamayor RO, C Pedrós-Alió, G Muyzer \& R Amann. 2002. Microheterogeneity in 16S rDNA-defind bacterial populations from a stratified planktonic environment is related to temporal changes and to ecological adaptations. Applied and Environmental Microbiology 68: 1706-1714.

Dumestre JF, EO Casamayor, R Massana \& C Pedrós-Alió. 2001. Changes in bacterial and archaeal assemblages in an equatorial river induced by the water eutrophication of Petit Saut dam reservoir (French Guiana). Aquatic Microbial Ecology 26: 209-221.

Ellis RJ, P Morgan, AJ Weightmann \& AJ Fry. 2003. Cultivation-dependent and -independent approaches for determining bacterial diversity in heavy-metal-contaminated soil. Applied and Environmental Microbiology 69: 3223-3230.

Escribano R. 1998. Population dynamics of Calanus chilensis in the Chilean Eastern Boundary Humboldt Current. Fisheries Oceanography 7(3/4): 245-251.

Escribano R \& P Hidalgo. 2000. Spatial distribution of copepods in the north of the Humboldt Current region off Chile during coastal upwelling. Journal of Marine Biology 80: 1-8.

Escribano R, V Marín, P Hidalgo \& G Olivares. 2002. Interacciones físico-biológicas en el ecosistema pelágico en la zona costera del área norte del sistema de la corriente de Humboldt. En: Castilla JC \& JL Largier (eds). The Oceanography and Ecology of the Nearshore and Bays in Chile, pp. 145-175. Ediciones Universidad Católica de Chile, Santiago.

González HE, G Daneri, D Figueroa, JL Iriarte, N Lefevre, G Pizarro, R Quiñónez, M Sobarzo \& A Troncoso. 1998. Producción primaria y su destino en la trama trófica pelágica y océano profundo e intercambio océano-atmósfera de $\mathrm{CO}_{2}$ en la zona norte de la corriente de Humboldt (23 S): posibles efectos del evento El Niño, 1997-98 en Chile. Revista Chilena de Historia Natural 71: 429-458.

Iriarte J, G Pizarro, V Troncoso \& M Sobarzo. 2000. Primary production and biomass of size-fractioned phytoplankton of Antofagasta, Chile (23-24 S) during pre-El Niño and El Niño 1997. Journal of Marine Systems 26: 37-51.

Iwamoto T, K Tani, K Nakamura, Y Suzuki, M Kitagawa, M Eguchi \& M Nasu. 2000. Monitoring impact of in situ biostimulation treatment on groundwater bacterial community by DGGE. FEMS Microbiology Ecology 32: 129-141.

Kenzaka T, N Yamaguchi, K Tani \& M Nasu. 1998. RNAtargeted fluorescent in situ hybridization analysis of bacterial community structure in river water. Microbiology 144: 2085 2093.

Lange C \& W Berger. 1993. Paleoclimatic significance of Santa Barbara laminated sediments: A history of upwelling and El Niño Events. EOS Transactions, American Geophysical Union 74(3): 372-375.
Lebaron P, P Servais, M Troussellier, C Courties, G Muyzer, L Bernard, H Schäfer, R Pukall, E Stackebrandt, T Guindulain \& J Vives-Rego. 2001. Microbial community dynamics in Mediterranean nutrient-enriched seawater mesoscosms: changes in abundances, activity and composition. FEMS Microbiology Ecology 34: 255-266.

Marín V \& G Olivares. 1999. Estacionalidad de la productividad primaria en bahía Mejillones del Sur(Chile): una aproximación proceso-funcional. Revista Chilena de Historia Natural 72: 629-641.

Marín V, L Delgado \& R Escribano. 2003. Upwelling shadows at Mejillones bay (northern Chilean coast): a remote sensing in situ analysis. Investigaciones Marinas 31(2): 47-55.

Marín V, L Rodríguez, L Vallejo, J Fuenteseca \& E Oyarce. 1993. Efectos de la surgencia costera sobre la productividad primaria primaveral de la Bahía Mejillones del Sur (Antofagasta, Chile). Revista Chilena de Historia Natural 66: 47-491.

Mullis KB \& FA Faloona. 1987. Specific synthesis of DNA in vitro via a polymerase-catalyzed chain reaction. Methods in Enzymology 155: 335-350.

Murray AE, CM Preston, R Massana, LT Taylor, A Blakis, K Wu \& EF DeLong. 1998. Seasonal and spatial variability of bacterial and archaeal assemblages in the coastal waters near Anvers Island, Antarctica. Applied and Environmental Microbiology 64: 2585-2595.

Myers RM, SG Fischer, LS Leman \& T Maniatis. 1985. Nearly all single base substitutions in DNA fragments joined to a GCclamp can be detected by denaturing gradient gel electrophoresis. Nucleic Acids Research 13: 3131-3145.

Muyzer G, EC de Waal \& AG Uitterlinden. 1993. Profiling of complex microbial populations by denaturing gradient gel electrophoresis analysis of polymerase chain reaction-amplified genes coding for $16 \mathrm{~S}$ rRNA. Applied and Environmental Microbiology 59: 695-700.

Muyzer G, T Brinkhoff, U Nübel, C Santegoeds, H Schäfer \& C Wawer. 1998. Denaturing gradient gel electrophoresis (DGGE) in microbial ecology. En: Akkermans ADL, JD van Elsas \& FJ de Brijn (eds) Molecular microbial ecology manual 3.4.4: 1-27. Kluwer Academic Publishers, Dordrecht.

Nelson DW \& LE Sommers. 1982. Total organic carbon and organic matter. En: Page AL, RH Miller \& DR Keeney (eds), Method of soil analysis. Part. 2: 539-579. American Society of Agronomy, Madison.

Nelson JD, S Boehme, C Reimers, R Sherrell \& L Kerkhof. 2008. Temporal patterns of microbial community structure in the Mid-Atlantic Bight. FEMS Microbiology Ecology 65: 484493.

Ortlieb L, R Escribano, R Follegati, O Zúñiga, I Kong, L Rodríguez, J Valdés, N Guzmán \& P Iratchet. 2000. Oceanclimatic changes during the last 2000 years in a hypoxic marine environment of Northern Chile $\left(23^{\circ} \mathrm{S}\right)$. Revista Chilena de Historia Natural 73: 221-242.

Rosado AS, GF Duarte, L Seldin \& JD van Elsas. 1998. Genetic diversity of nifH gene sequences in Paenibacillus azotofixana strains and soil samples analyzed by denaturing gradient gel 
electrophoresis of PCR-amplified gene fragments. Applied and Environmental Microbiology 64: 4650-4657.

Sahm K \& UR Berninger. 1998. Abundance, vertical distribution, and community structure of benthic prokaryotes from permanently cold marine sediments (Svalbard, Arctic Ocean). Marine Ecology Progress Series 165: 71-80.

Santegoeds CM, TG Ferdelman, G Muyzer \& D Beer. 1998. Structural and functional dynamic of sulfate reducing populations in bacterial biofilms. Applied and Environmental Microbiology 64: 3731-3739.

Schäfer H, L Bernard, CC Courties, P Lebaron, P Servais, $R$ Pukal, E Stackebrandt, M Trousselier, T Guindulain, J Vives-Rego \& G Muyzer. 2001. Microbial community dynamics in Mediterranean nutrient-enriched seawater mesocosms: changes in the genetic diversity of bacterial populations. FEMS Microbiology Ecology 34: 243-253.

Sobarzo M \& D Figueroa. 2001. The physical structure of a cold filament in a Chilean upwelling zone (Peninsula de Mejillones, Chile, $\left.23^{\circ} \mathrm{S}\right)$. Deep Sea Research Part I: Oceanographic Research Papers 48: 2699-2726.

Strub P, J Mesias, V Montecinos, J Rutllant \& S Marchant. 1998. Coastal oceanic circulation off western South America. En: Robinson A \& K Birnk (eds) The Sea, pp. 273-314. John Wiley, Nueva York.

Summerhayes C, K Emeis, M Angel, R Smith \& B Zeitzschel. 1995. Upwelling in the ocean: modern processes and ancient records. Environmental Sciences Research Report 18: 1-37.

Thomas A, J Blanco, M Carr, P Strub \& J Osses. 2001. Satellitemeasured chlorophyll and temperature variability off northern Chile during the 1996-1998 La Nina and El Nino. Journal of Geophysical Research 106(C1): 899-915.

Traian B, B van Breukelen, M Braster, N van Straalen \& W Röling. 2008. Spatial heterogeneity in sediment-associated bacterial and eukaryotic communities in a landfiell leachatecontaminated aquifer. FEMS Microbiology Ecology 65: 534543.
Tsai YL \& BH Olson. 1991. Rapid method for direct extraction of DNA from soil and sediment. Applied and Environmental Microbiology 57: 1070-1074.

Valdes J, D Roman, G Alvarez, L Ortlieb \& M Guinez. 2008. Metals content in surface waters of an upwelling system of the northern Humboldt Current (Mejillones Bay, Chile). Journal of Marine Systems 71: 18-30.

Valdes J, G Vargas, A Sifeddine, L Ortlieb \& M Guinez. 2005. Distribution and enrichment evaluation of heavy metals in Mejillones Bay $\left(23^{\circ} \mathrm{S}\right)$, Northern Chile: Geochemical and statistical approach. Marine Pollution Bulletin 50(12): 15581568.

Van Hannen EJ, M Veninga, J Bloem, HJ Gons \& HJ Laanbroek. 1999. Genetic changes in the bacterial community structure associated protistan grazers. Archaeal Hydrobiology 145: 25-38.

Vargas G. 2002. Interactions ocean-atmosphère au cours des derniers siècles sur la côte du Désert d'Atacama, analyse multiproxies des sédiments laminés de la Baie de Mejillones $\left(23^{\circ} \mathrm{S}\right)$. $\mathrm{PhD}$ Thesis, Universitè Bordeaux I, France, $290 \mathrm{pp}$.

Wagner M, RAmann, H Lemmer \& KL Schleifer. 1993. Probing activate sludge with oligonucleotides specific for Proteobacteria: inadequacy of culture-dependent methods for describing microbial community structure. Applied Environmental Microbiology 59: 1520-1525.

Ward N, FA Rainey, B Goebel \& E Stackebrandt. 1995. Identifying and culturing the unculturables: a challenge for microbiologist: En: Alsopp D, RR Colwell \& DL Hawksworth (eds). Microbial Diversity and Ecosystem Function, pp. 89110. CAB International, Wallingford. 